\title{
Embedded mobile crowd service systems based on opportunistic geological grid and dynamical segmentation
}

\author{
Chunhua Dong ${ }^{1,2^{*}}$, Li Wang ${ }^{2}$ and Kunming Zhao ${ }^{2}$
}

\begin{abstract}
In order to solve these problems such as the demand of geographic information service and the short life of the embedded system, as well as network collapse, and so on, the embedded mobile crowd service systems based on opportunistic geological grid and dynamical split was proposed. Firstly, based on the characteristics of geographical spatial information resources and service time series, a mobile geographic crowd service system was established for providing the sensing data with the mobile geographic crowd service model. Then, according to the embedded equipment complex data of the geographic crowd service system, and the relationship between the geography information service object and the user, the embedded system was proposed based on the opportunity geological grid. Finally, the optimization of the geographic crowd system was realized by the dynamic segmentation of the opportunity geographic grid. The experiment results of the equipment utilization, the life cycle of the crowd network, user satisfaction, and control complexity show that the proposed scheme is more suitable for the embedded network geographic information system.
\end{abstract}

Keywords: Geographic information system, Embedded mobile crowd service systems, Opportunistic geological grid, Dynamical segmentation

\section{Introduction}

With the rapid development of embedded system and mobile communication network [1], the above technologies are applied in the description of the geological environment, the location of the landmarks, and the business of the exploration information. The embedded system and mobile communication network technology are used to satisfy the core requirements of geographic information system, such as geological information, map data, and multimedia data [2], which has been the research hot issue in the academic field [3].

Galib Krdzalic et al. [4] designed a simple, modular, and universal architecture for an electric vehicle charging cable between industry and automobile applications. The static priority scheduling of recurring real-time tasks was studied by focusing on the non-preemptive uniprocessor case and obtaining schedule theoretic results in article [5]. R. R. Aguiar et al. [6] researched the experimental

\footnotetext{
* Correspondence: dongchhua@sina.com

${ }^{1}$ China University of Mining and Technology (Beijing), Beijing 100083, China ${ }^{2}$ Tianjin Chengjian University, Tianjin 300384, China
}

investigation and the mathematical modeling of the impact force behavior in a vibro-impact system. The prototype system was developed by Chenshu $\mathrm{Wu}$ et al. [7] for conducting comprehensive experiments in both crowded urban and spacious suburban areas. An incentive mechanism was proposed by Yutian Wen et al. [8] based on a quality-driven auction, which is specifically for the mobile crowd sensing system. In an article [9], a crowd-powered sensing system was presented for crossspace public information reposting, tagging, and sharing.

The stochastic reliability of smart grids and opportunistic energy users was studied, and the procurement of energy supply from both conventional generations was investigated [10]. The feasibility of OR use in power line communications-access network-was investigated and a customized opportunistic routing was proposed with static geographical information [11]. An energy efficient and reliable medium access control protocol for cognitive sensor networks was designed in [12]. An operator method was proposed in [13] for calculating the semi classical asymptotic form with the energy splitting value

\section{Springer}


in the general case of tunneling. A fully explicit was proposed and analyzed in [14] by computationally inexpensive integrator and splitting the orthogonal projector onto the tangent space of the low-rank manifold. A procedure was presented [15] for leading to efficient splitting schemes of explicitly time-dependent partitioned linear differential equations after discretizing the certain partial differential equations in space.

On the basis of the above research results, the problems such as the changes of network environment and the short life cycle of geographic information system (GIS) were studied. Our main research results are as follows: (1) the platform of the embedded system was proposed. (2) The embedded system is proposed. (3) The optimization of the geographic crowd service system was realized by dynamic partitioning.

The rest of the paper is organized as follows. Section 2 describes the mobile crowd sensing network for power grid management. In Section 3, we discussed the mobile service aware opportunistic embedded system. In Section 4, we proposed the grid intelligent management of embedded systems. The performance analysis of embedded system has been shown in Section 5. Finally, the conclusion is given in Section 6 .

\section{Mobile geographic crowd service model}

Based on mobile embedded devices, mobile communication platform, and mobile applications, the mobile geographic crowd service system was established based on geographic information resources and time series. The system defines the time series as a reference, uses the mobile embedded device to perceive the geographic spatial information, and provides reliable geographic information service for the public and industry users through the mobile communication platform. The core of system is the distributed, embedded, and synchronous heterogeneous geographic crowd service. The system satisfies the diversity requirements and dynamic topology of mobile communication network through the embedded organization and active data management of the geographic information resources. The system architecture is shown in Fig. 1.

Based on the architecture shown in Fig. 1, the mobile geographic crowd service system includes the following functions:

(1) Share the information service resources of the diversity in the time and space.

(2)Construct dynamic adaptive geographic information based on the active mobile crowd service geographic information service with space and time.

(3) The connection and integration of real-time geographic information and services based on mobile embedded devices.
The time series A of system, spatial location information matrix $\mathrm{B}$, and the service information data sequence $\mathrm{X}$ satisfy the linear Eq. (1).

$$
\left\{\begin{array}{c}
A=\left[\begin{array}{lll}
a_{1} & \cdots & a_{n}
\end{array}\right] \\
B=\left[\begin{array}{lll}
b_{11} & \cdots & b_{1 n} \\
b_{21} & \ddots & b_{2 n} \\
b_{m 1} & \cdots & b_{m n}
\end{array}\right] \\
X=\left[\begin{array}{lll}
x_{1} & \cdots & x_{n}
\end{array}\right]^{T} \\
X=\frac{\alpha A+\gamma B}{\sqrt{A^{2}+B^{2}}}
\end{array}\right.
$$

Here, $\alpha$ denotes the real-time weight of time series. $\gamma$ denotes the credibility of geographic location information.

There are the following relationship between the $\mathrm{k}$ satisfaction of active geographic crowd service and k-1 satisfaction as shown in Eq. (2).

$$
x^{k}=\sqrt{\alpha^{2}+\gamma^{2}}\left[\begin{array}{cccc}
a_{1} b^{k-1}{ }_{11} & a_{1} b^{k-1}{ }_{12} & \cdots & a_{1} b^{k-1}{ }_{1 n} \\
a_{2} b^{k-1}{ }_{21} & a_{2} b^{k-1}{ }_{22} & \cdots & a_{1} b^{-1}{ }_{2 n} \\
\vdots & \ddots & \ddots & \vdots \\
a_{m} b^{k-1}{ }_{m 1} & a_{m} b^{k-1}{ }_{m 2} & \cdots & a_{m} b^{k-1}{ }_{m n}
\end{array}\right] x^{k-1}
$$

Gauss elimination of the formula (2) is simplified as formula (3).

$$
x^{k}=\frac{1}{a_{k} b_{k, k}}\left(a_{k-1} b_{k-1, k-1}-\frac{\alpha}{\gamma} \sum_{i=1}^{k-1} a_{i}^{k-1} x_{i}^{k-1}\right)
$$

Here, the time series and spatial location information of the $\mathrm{k}$ service can be obtained by the actual measurement.

On the basis of linear Eq. (1), a series of linear iterative equations are set up for the mobile geographic crowd service, as shown in formula (4).

$$
\left\{\begin{array}{l}
x^{k}=f(A) \gamma x^{k-1}+h(B)^{\alpha} \\
f(a)=f(a-1) \bar{x} \\
h(b)=h(b-1) f(\bar{x}) \\
\lim _{k \rightarrow \infty} x_{k}=\|\bar{x}\|^{2}
\end{array}\right.
$$

Here, the time series are normalized by the function $f(a)$. The geographic information data is processed by $h(b), \bar{x}$ represents the average value of the mobile geographic cluster intelligence service that has been recognized by the user.

After multiple iterations, the error between the actual service value and the user's requirement can be obtained by the formula (5).

$$
\left\{\begin{array}{l}
\left\|x_{k}-\tilde{x}_{k}\right\| \leq\|\bar{x}\|,|\alpha-\gamma|=0 \\
\left\|x_{k}-\tilde{x}_{k}\right\| \leq \frac{1}{\bar{x}}\left|\sum_{i=1}^{k} x_{i}\right|,|\alpha-\gamma| \neq 0
\end{array}\right.
$$

Here, $\tilde{x}_{k}$ represents the actual user base service demand value. $|\alpha-\gamma|=0$ denotes the geographic information 


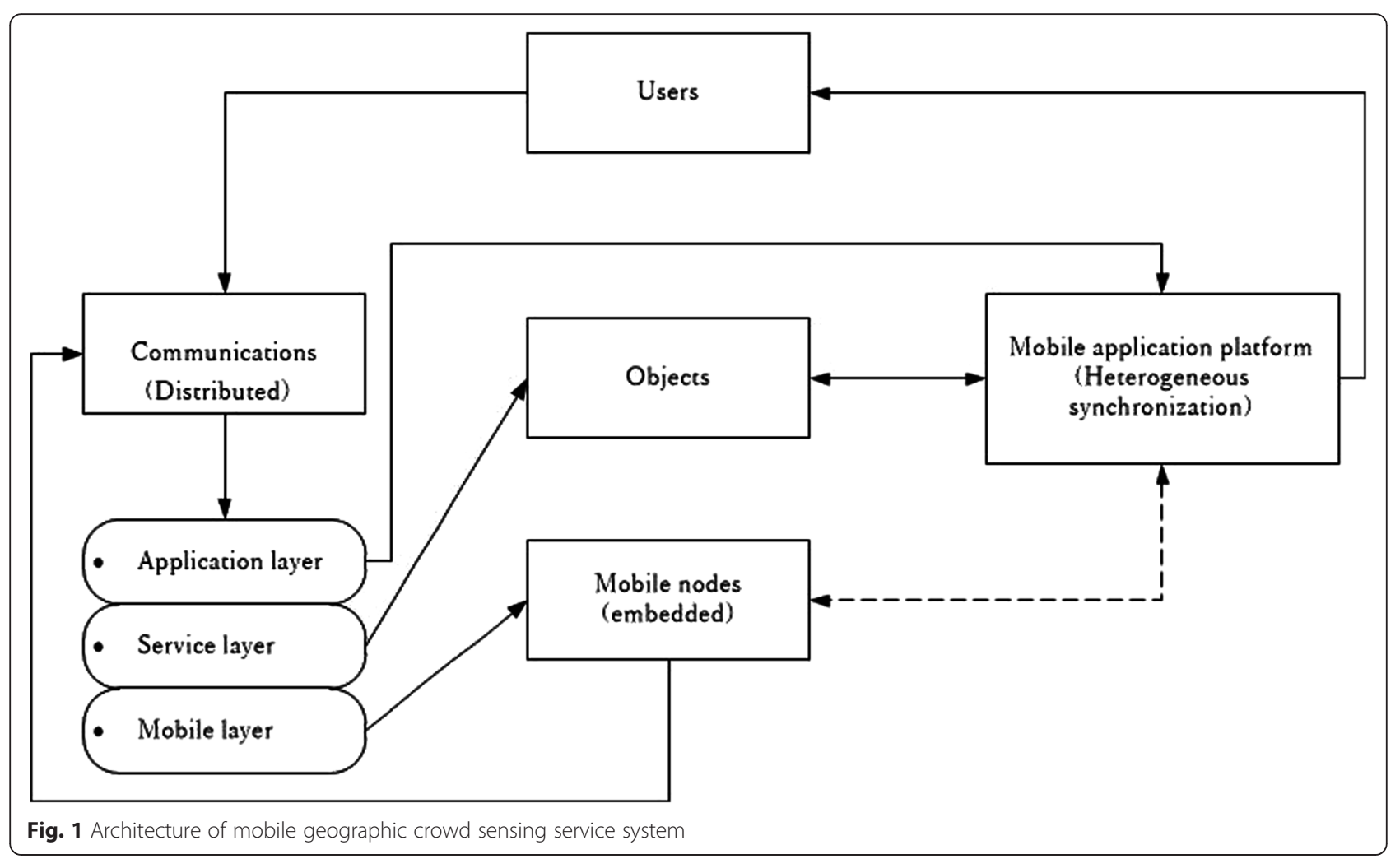

group crowd service has a high degree of overlap. However, there is a great inhibition to the iterative speed and the convergence speed in time and space.

Therefore, the processing method of formula (6), as far as possible, to keep the time and space on the geographical information to maintain the orthogonally, to satisfy the orthogonal is shown in formula (7).

Here, $\vec{x}_{k}$ denotes the optimizing value of $\mathrm{k}$ mobile crowd service.

Hence, a comprehensive mobile geographic crowd service set of $n$ users is obtained from the mobile application platform, as shown in formula (8).

$$
S\left(x_{1}, \cdots, x_{n}\right)=\frac{\left|f(A)-h\left(\sum_{i=1}^{n} b_{i i}\right)\right|}{x_{n}-x_{m}}
$$

\section{Embedded GIS based on the opportunity geological grid}

The embedded device perception complex data of the geographic crowd service system includes the geographic service description information, the spatial position of the service object, and the correlation between the service network and the user. The complex data has the following features:
(1) Complexity sequence.

This complexity is the most important feature of embedded data sequence. It is a complex performance. The complexity of the sequence of $S_{C}$ includes service description complexity $\mathrm{SD}_{\mathrm{C}}$, service object space location complexity $\mathrm{SS}_{\mathrm{C}}$, service object dynamic form complexity $\mathrm{SF}_{\mathrm{C}}$, and computational complexity $C_{\mathrm{C}}$. Among them, it is the key factor to restrict the performance of the system, which can be described by the formula (9).

$\left\{\begin{array}{l}S_{\mathrm{C}}=\left[\begin{array}{l}\mathrm{SD}_{\mathrm{C}} \\ \mathrm{SS}_{\mathrm{C}} \\ \mathrm{SF}_{\mathrm{C}} \\ C_{\mathrm{C}}\end{array}\right]\left[\begin{array}{l}W_{1} \\ W_{2} \\ W_{3} \\ W_{4}\end{array} W_{1}+W_{2}+W_{3}+W_{4}=1\right.\end{array}\right.$

Here, $W_{1}, W_{2}, W_{3}$, and $W_{4}$ represent the weight of service description complexity $\mathrm{SD}_{\mathrm{C}}$, service object space location complexity $\mathrm{SS}_{\mathrm{C}}$, service object dynamic form complexity $\mathrm{SF}_{\mathrm{C}}$, and computational complexity $C_{C}$, respectively. In order to maintain the consistency of the multiuser geographic knowledge service, the sum of all the complexity is 1 .

(2)Service satisfaction.

The geographic service description sequences of complex data include the features of real objects, the 
service structure, and the service environment. These factors are dynamic changes, and the conversion process is very complex. In order to quantify the mapping relationship between the swarm intelligence and the service object and the flow service process, the service satisfaction parameter SSA is given. The parameters are obtained by sampling $S_{\mathrm{AM}}$, quantization $Q_{\mathrm{UA}}$, and discrete $D_{\mathrm{SC}}$ after abstract $A_{\mathrm{BS}}$ processing, as shown in formula (10).

$$
\left\{\begin{aligned}
S_{\mathrm{SA}} & =S_{\mathrm{AM}} \oplus Q_{\mathrm{UA}} \oplus D_{\mathrm{SC}} \oplus A_{\mathrm{BS}} \\
S_{\mathrm{AM}} & =\int_{i=1}^{t} x(t) \\
Q_{\mathrm{UA}} & =\sum_{i=0}^{t} h(i) e^{-W_{1} S_{\mathrm{SC}}} \\
D_{\mathrm{SC}} & =\sqrt{\frac{1}{t} \sum_{i=1}^{t} y(i)^{S_{\mathrm{C}}}} \\
A_{\mathrm{BS}} & =\left.x(t)\right|_{t} e^{-n S_{\mathrm{C}}} \phi(t)
\end{aligned}\right.
$$

Here, $x(t)$ is a representation of the transmitted signal sequence. $y(t)$ is a sequence of received signals. $t$ represents the geographic information network as a service object to provide a geographic information delay. $N$ indicates the sequence length. $\phi(t)$ is the signal abstract processing function after discrete progress.

(3) Polymorphism of crowd network.

The shape and topology of the geographical crowd network have different precision and performance form in different service flow and service object. For the service process of the same service object, the geographic information of the service target has different forms with the change of time and space and has obvious difference. So, complex data must have the characteristics of self correlation and can be obtained by formula (11) to characterize the self-correlation of the group $\mathrm{CS}_{\mathrm{PO}}$.

$\left\{\begin{array}{l}\mathrm{CS}_{\mathrm{PO}}=\left\{\begin{array}{l}\mathrm{PO}_{1}, K=1 \\ \vdots \\ \mathrm{PO}_{M}, K=M\end{array}\right. \\ K=\frac{\sum_{i=1}^{\sqrt{M}} x(t)^{i}}{M}\end{array}\right.$

Here, $\mathrm{PO}$ indicates the form of crowd net. $K$ is a polymorphic weight value. $M$ indicates the number of crowd network clusters.

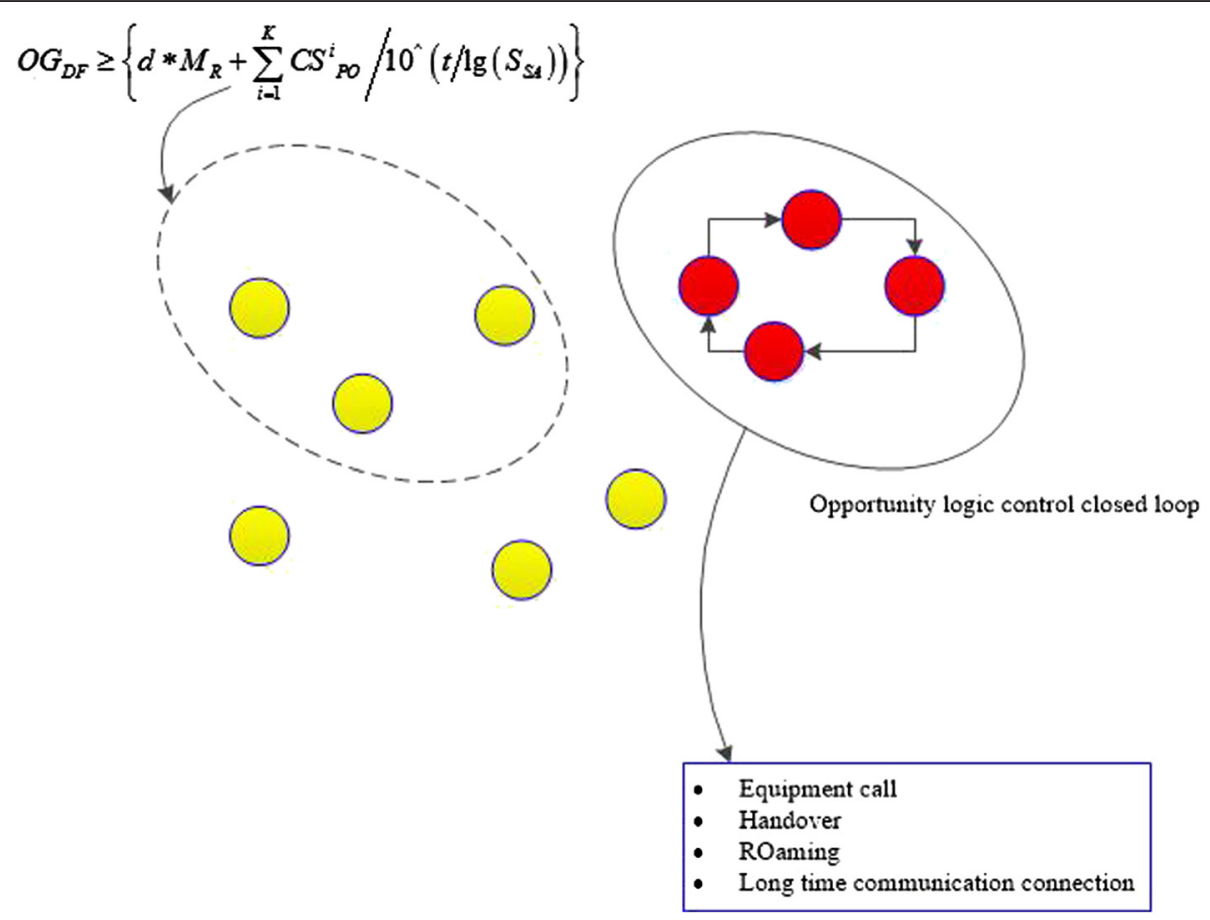

Fig. 2 Opportunity geological grid model 
(4) The mutual relations between the geographic service and the crowd network.

In order to provide services to more users in the environment of linear extension and spatial dynamics, the sequence of services provided by the geographic knowledge network must have significant correlation with each other. The correlation of the correlation between time series, spatial sequence, frequency sequence and service sequence must be positive. Therefore, the complex data of geographical crowd system is a multi-scale and multiform and weak cross-correlation. The mutual relationship can be obtained by the matrix operation shown in the formula (12).

$M_{R}=\int_{t=1}^{\infty} f_{t} S_{t} d t$

According to the characteristics of the embedded devices complex data in the crowd service system, the system characteristics such as the opportunity of call, switch, roaming, and longtime communication connection, the opportunity grid was established with multiple embedded devices in the crowd networks, as shown in Fig. 2.

The formula (13) gives the logical control of the opportunity geological grid. The logical opportunity control would be started up when the inequality satisfied the formula (13).

$\mathrm{OG}_{\mathrm{DF}} \geq\left\{d * M_{R}+\sum_{i=1}^{K} \mathrm{CS}_{\mathrm{PO}}^{i} / 10^{\wedge}\left(t / \lg \left(S_{\mathrm{SA}}\right)\right)\right\}$

This opportunity grid is driven by the geological information within the service area and can realize the seamless handover and provide reliable communication of the geographic information service by opportunistic control. Opportunity logic control and wireless transparent interface are realized through the embedded equipmentindependent computing and full duplex point-topoint communication such as shown in Fig. 3.

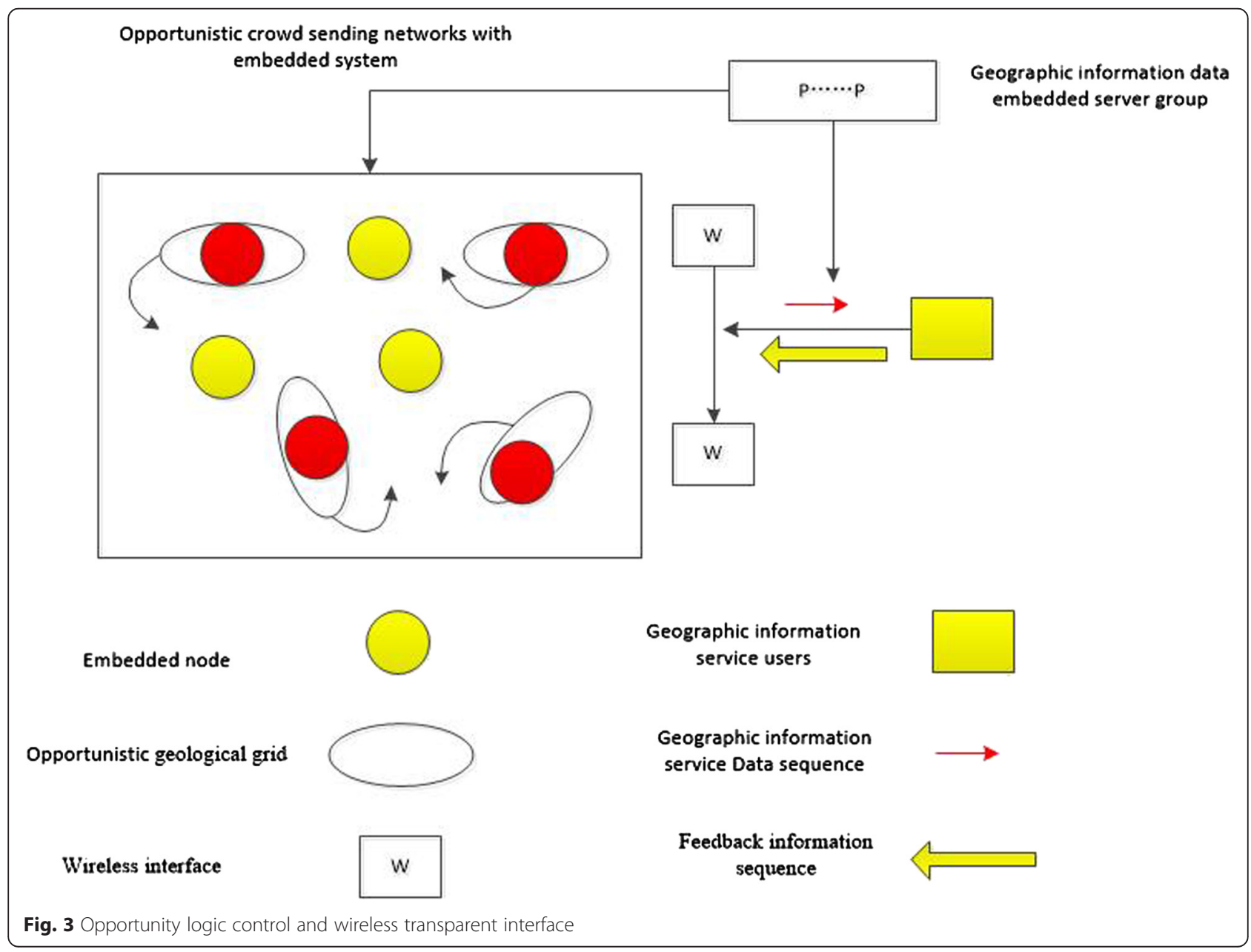




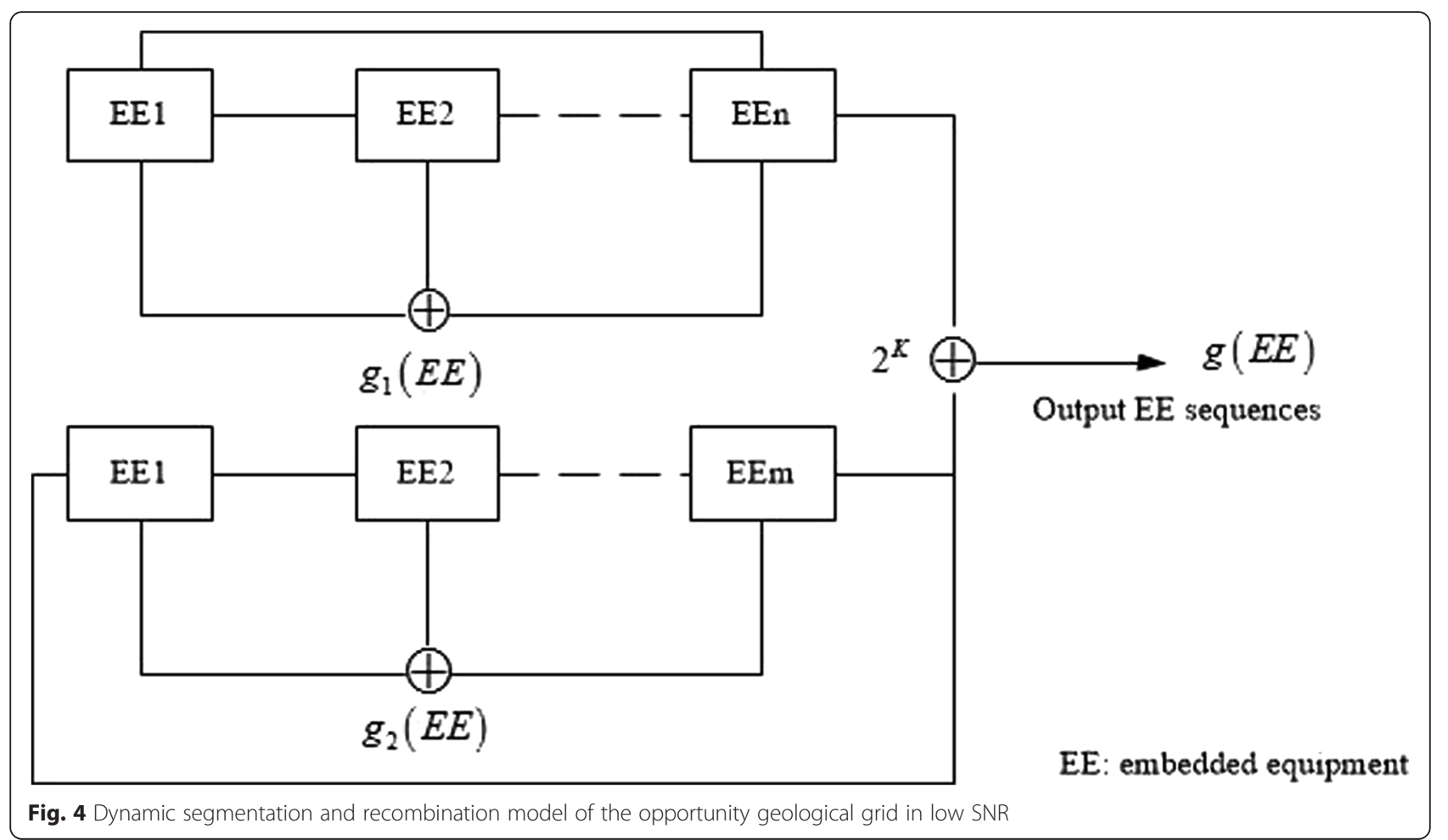

\section{Embedded mobile opportunity crowd service mechanism based on dynamic segmentation}

The opportunity geological grid should be dynamically segmented for constituting a new opportunity geological grid with geographic information services, according to the time, space, frequency, geomorphology, geological, location, and other information of the dynamic change. So, a new geographic knowledge service system would be formed. The following is a dynamic segmentation and processing procedure of the opportunity geological grid.

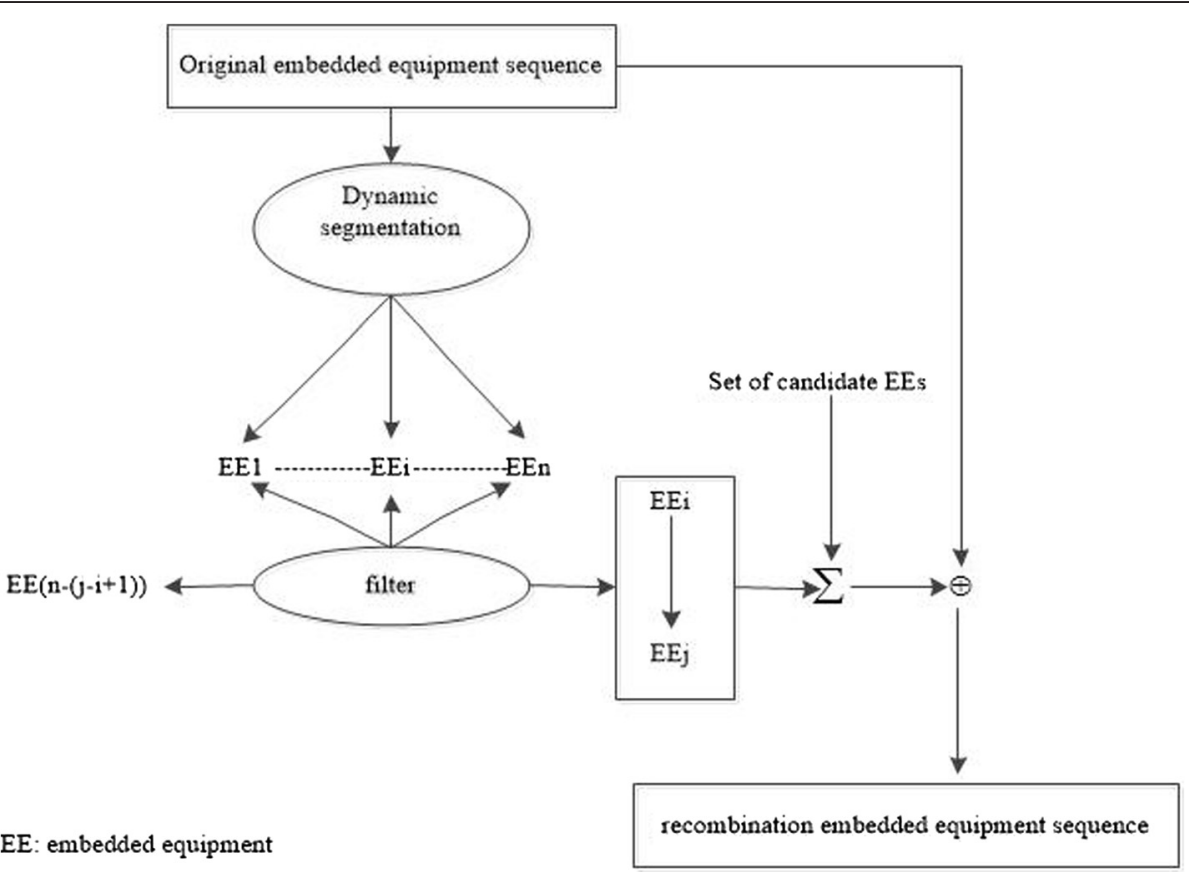

Fig. 5 Dynamic segmentation and recombination model of the opportunity geological grid based on quality of service 
First, the dynamic segmentation would be started when the signal to noise ratio is lower than the threshold value. The opportunity geological grid would be divided into two parts. One part includes the embedded device whose signal to noise ratio is higher than the threshold value. The other part includes the independent embedded devices. The embedded separation devices could join other opportunity geological grid when they have been adjusted and updated. The original embedded equipment and the neighbor devices would reconstruct the current opportunity geological grid. The signal to noise ratio of the neighbor devices should be higher than the threshold values, and the mutual correlation is weak.

The opportunity geological grid should be dynamic segmented and reorganized in the geographic crowd service system. $g_{1}(\mathrm{EE})$ is composed of an embedded system with an embedded system in the prior opportunity geological grid. The polynomial $g_{2}(\mathrm{EE})$ is the sequence of embedded devices. The reorganization of the opportunity geological grid equipment sequence $g(\mathrm{EE})$ can be obtained after $2 \mathrm{~K}$ iterations of the XOR operation, which is shown in Fig. 4.

Second, when the opportunity geological grid would be dynamically segmented when the quality of the geographic information service is lower than the threshold value.

The embedded devices of the opportunity geological grid would be divided into two parts. One part includes the embedded equipment that has abundant remaining resources. The other part has the failure embedded equipment, which cannot continue to provide geological services because of the resource exhaustion. These embedded devices would be separated into the independent embedded devices, which could join other opportunity geological grid when they have been adjusted and updated. The original embedded equipment and the

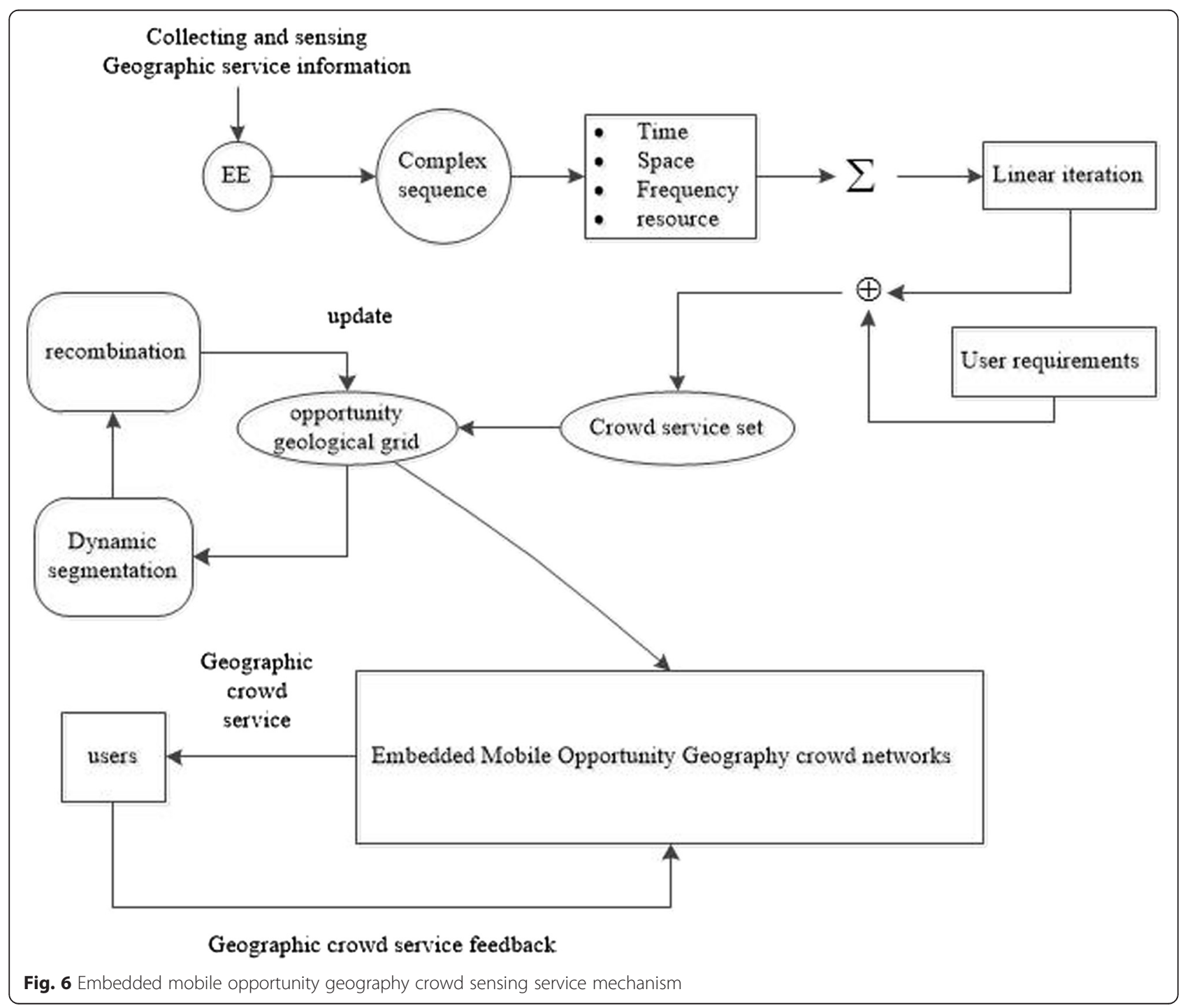


Table 1 Experiment settings

\begin{tabular}{llll}
\hline Parameters & Value & Descriptions of system & Value \\
\hline Number of embedded equipment & 100 & Development of algorithm & In embedded equipment and control by hardware \\
Number of geography services & 30 & Protocol of wireless communication & TS-SCDMA + IEEE $802.11 \mathrm{~g}$ \\
Number of geography information services & 20 & Operating system of servers & Ubuntu 13.10 \\
Scale & $5 \mathrm{~km}^{2}$ & Interval of opportunity geological grid & {$[5,10]$} \\
Type of database & MySQL & Geographic information service type & Location information, geological information, map \\
& & & information and multimedia information \\
\hline
\end{tabular}

neighbor devices would reconstruct the current opportunity geological grid. The signal to noise ratio of the neighbor devices should be higher than the threshold values, and the mutual correlation is weak, as shown in Fig. 5.

Embedded mobile opportunity geographic knowledge service mechanism is described in Fig. 6. The specific process has the following characteristics:

(1) The embedded mobile device could percept the geographic service information and generate a complex sequence.

(2) The complex sequence includes the time, space, frequency diversity, and resource management.

(3) The linear iterative processing scheme would get the data sum.

(4) The cluster intelligence service cluster would be generated in this process.

(5)Dynamic segmentation, reorganization, and renewal of the opportunity geological grid would be completed in this process.

(6) The embedded geographic group would be controlled opportunistically.

(7) The geographic service information would be sent to the user.

(8) A crowd service feedback would be sent by the users.

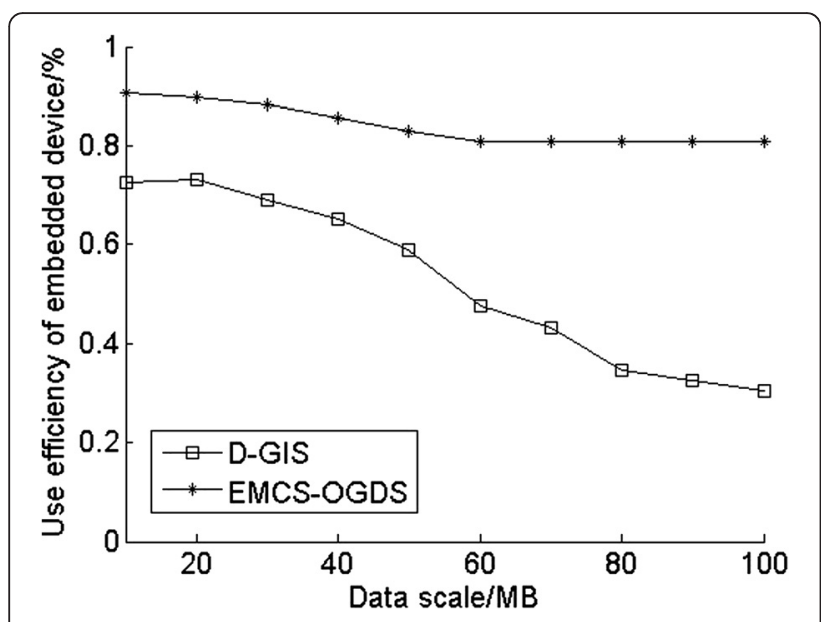

Fig. 7 Use efficiency of embedded device

\section{Performance analysis and verification}

Based on embedded devices, the performance of mobile intelligent network in the application of geographic information services is generally focused on the following aspects:

(1) The use efficiency of embedded devices. This parameter can be obtained by the perception of the embedded devices and the scale of the geographic information data.

(2) The life cycle of the mobile group. The parameters of the users are used to send a geographic information service request time to the group.

(3) Embedded system control complexity. That means the time complexity, space complexity, and computational complexity.

(4) Customer service quality satisfaction. This parameter can be obtained by means of statistical user feedback information.

In order to analyze the performance of the proposed system and its control mechanism, we design the experiment demonstrated in Table 1. We compared the four aspects performance EMCS-OGDS and distributed GIS (D-GIS). The results are shown in Figs. 7, 8, and 9.

Figure 7 shows the efficiency of the use of embedded devices. It is found that the embedded equipment of

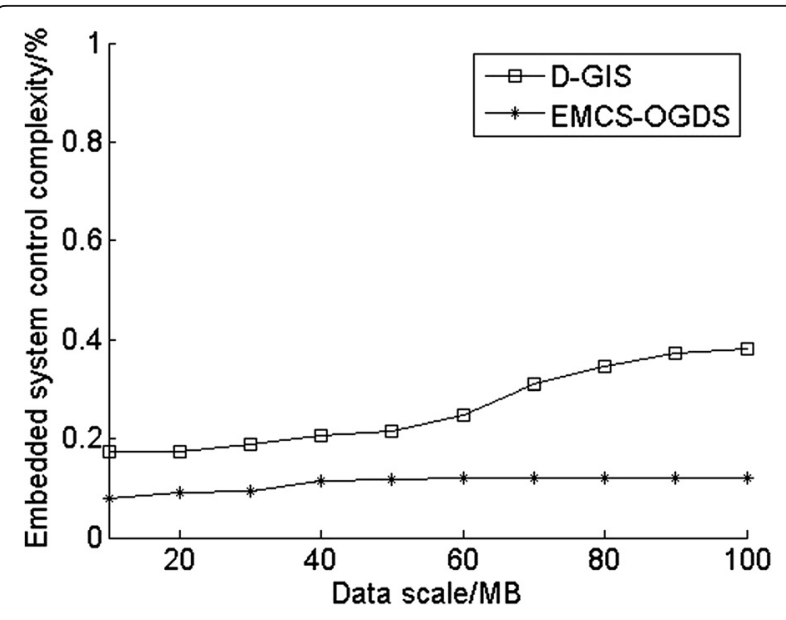

Fig. 8 Embedded system control complexity 


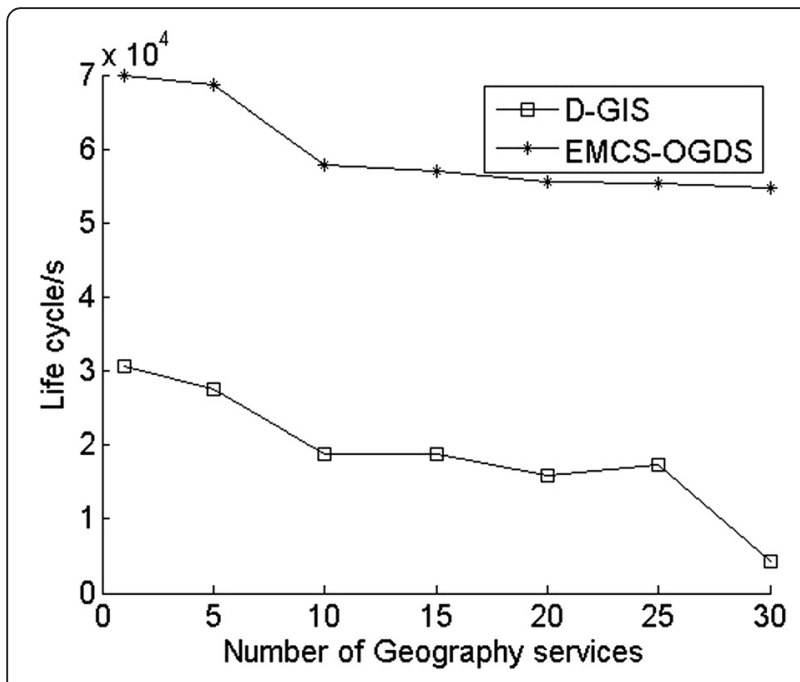

Fig. 9 Life cycle of Mobile Crowd Sensing Networks

EMCS-OGDS is a unit of opportunity, which has high accuracy of geographic information data perception ability. Moreover, the opportunity control logic is embedded in the hardware chip of mobile devices, and the working process of the device is controlled by the dynamic change of environment. In the same way, the embedded system has obvious advantages in time complexity, space complexity, and computational complexity, as shown in Fig. 8.

Figure 9 shows the life cycle of the mobile crowd network under the control of the two mechanisms. We found that the life cycle of D-GIS significantly shortened when the number of user's geographic information service request is higher than 15 , which is because that the resources of large-scale-embedded devices fast collapse. The network breakdown and customer service satisfaction gradually reduced, see Fig. 10. However, EMCSOGDS has the dynamic segmentation of extreme value, which can optimize the opportunity to advance the

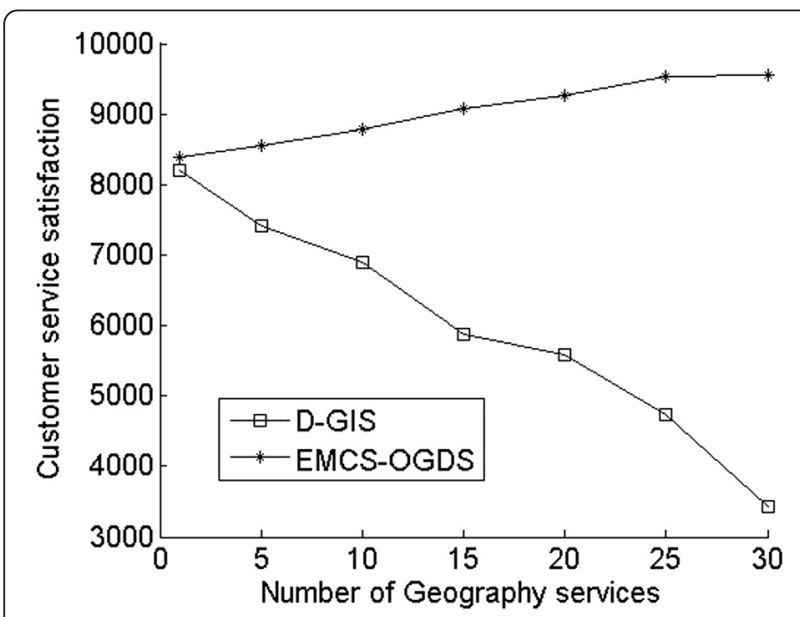

Fig. 10 Customer service satisfaction geological grid, to extend the life cycle of the swarm intelligence network and to ensure that users get high quality service.

\section{Conclusions}

In this paper, based on the geographic information service requirements and mobile embedded systems, we analyzed and studied the application characteristics of mobile crowd network in geographic information system with embedded devices. Firstly, the geographic information service system is established by combining the geographic information resources and time linear sequences. And then, opportunistic geological grid system was proposed based on the characteristics of geographic information service users' demand and the embedded devices. Finally, according to the dynamic information of time, space, frequency, geomorphology, geology, location, etc., the new opportunity geological grid would be reconstructed with dynamical segmentation, which would update the embedded mobile crowd service systems. Experimental results show that the proposed geographic information system has obvious advantages in resource utilization, network robustness, user satisfaction, and control complexity.

\section{Competing interests}

The authors declare that they have no competing interests.

\section{Acknowledgements}

This work is supported in part by the National Major Research Equipment Development Project (ZDYZ2012-1-05) and National Natural Science Fund (51508372).

Received: 14 November 2015 Accepted: 16 December 2015

Published online: 26 December 2015

References

1. EK Çetinkaya, MJF Alenazi, AM Peck et al., Multilevel resilience analysis of transportation and communication networks. Telecommun. Syst. 60(4), 515-537 (2015)

2. Y Xiao, Q Huang, K Wu, Understanding social media data for disaster management. Nat. Hazards 79(3), 1663-1679 (2015)

3. G Barrientos, L Catella, F Oliva, The spatial structure of lithic landscapes: the late Holocene record of East-Central Argentina as a case study. J. Archaeol. Meth. Theor. 22(4), 1151-119 (2015)

4. G Krdzalic, A Driss, Software architecture without AUTOSAR general template for embedded systems. Atzelektronik Worldwide 2014, Volume 9, Issue 1, pp 20-23

5. C Erbas, AD Pimentel, S Cerav-Erbas, Static priority scheduling of eventtriggered real-time embedded systems. Formal Meth. Syst. Des. 30(1), 49 (2007)

6. RR Aguiar, HI Weber, Mathematical modeling and experimental investigation of an embedded vibro-impact system. Nonlinear Dynam. 65(3), 317-334 (2011)

7. C Wu, Z Yang, Y Xu et al., Human mobility enhances global positioning accuracy for mobile phone localization. IEEE Trans. Parallel Distr. Syst. 26(1), 131-141 (2015)

8. Y Wen, J Shi, Q Zhang et al., Quality-driven auction-based incentive mechanism for mobile crowd sensing. IEEE Trans. Veh. Technol. 64(9), 4203-4214 (2015)

9. B Guo, H Chen, Z Yu et al., FlierMeet: a mobile crowd sensing system for cross-space public information reposting, tagging, and sharing. IEEE Trans. Mob. Comput. 14(10), 2020-2033 (2015)

10. M He, S Murugesan, J Zhang, A multi-timescale scheduling approach for stochastic reliability in smart grids with wind generation and opportunistic demand. IEEE Trans. Smart Grid 4(1), 521-529 (2013) 
11. S-G Yoon, S Jang, Y-H Kim et al., Opportunistic routing for smart grid with power line communication access networks. IEEE Trans. Smart Grid 5(1), 303-311 (2014)

12. A Aijaz, S Ping, MR Akhavan et al., CRB-MAC: a receiver-based MAC protocol for cognitive radio equipped smart grid sensor networks. IEEE Sensors J. 14(12), 4325-4333 (2014)

13. EV Vybornyi, Energy splitting in dynamical tunneling. Theor. Math. Phys. 181(2), 1418-1427 (2014)

14. C Lubich, IV Oseledets, A projector-splitting integrator for dynamical low-rank approximation. BIT Numer. Math. 54(1), 171-188 (2014)

15. S Blanes, F Casas, A Murua, Splitting methods in the numerical integration of non-autonomous dynamical systems. Revista de la Real Academia de Ciencias Exactas, Fisicas y Naturales. Serie A. Matematicas 106(1), 49-66 (2012)

\section{Submit your manuscript to a SpringerOpen ${ }^{\circ}$ journal and benefit from:}

- Convenient online submission

- Rigorous peer review

- Immediate publication on acceptance

- Open access: articles freely available online

- High visibility within the field

- Retaining the copyright to your article

Submit your next manuscript at $>$ springeropen.com 